\title{
Using knowledge mapping to support knowledge translation in health organizations
}

\begin{abstract}
Lavkush
National Documentation Centre, National Institute of Health and Family Welfare, Munirka, New Delhi-110067, India.

Accepted 18 May, 2011

This article briefly discusses knowledge translation and knowledge mapping. It focuses on the use of knowledge mapping as a technique to support knowledge translation in health organizations in order to bridge the gap between knowledge generated and action for health. This paper explores the possibility for integrating knowledge mapping with conceptual framework so that it can help understanding many complex processes, resources and people involved in health system.
\end{abstract}

Key word: Knowledge mapping, knowledge translation, health organizations.

\section{INTRODUCTION}

Knowledge translation program was founded in the year 2000 by the Department of Medicine, Family and Community Medicine, Health Policy Management and Evaluation, Public Health Sciences, through funding from the Academic Priorities fund, University of Toronto. The process was developed in response to the gap between research evidence and clinical practice. Initiatives were needed to facilitate interaction between researchers and policy-makes to foster greater use of research findings and evidence in policy-making and to narrow the "knowdo-gap".

The Canadian Institute of Health Research (CIHR) has referred to knowledge translation as the process of supporting the uptake of health research in a manner that improves the health and health care of Canadians through improved understandings, processes, sciences, products and systems.

A vast amount of global investments in health research generated pool of knowledge that is underused and not translated rapidly enough into new health policies, products, services and outcomes. Knowledge translation has come at a time when the gap between what is known and what gets done (the known -do gap) is highlighted by shortfalls in equity and quality in health services.

\section{KNOWLEDGE MAPPING}

Knowledge mapping is a process by which organizations can identify and categories knowledge assets within their organization-people, processes, content, and technology. It allows an organization to fully leverage the existing expertise resident in the organization, as well as identify barriers and constraints to fulfilling strategic goals and objectives. It is constructing a roadmap to locate the information needed to make the best use of recourses, independent of source or form (Vestal, APQC, 2002).

\section{What does knowledge translation stand for?}

Knowledge translation can be used as a noun or a verb. It is described a structured process that results in scientifically research evidence being used to improve 
the outcomes of professional practice. Knowledge can be created without science and knowledge translation is not research; it moves from responding to curiosity to focusing on purpose and problems solving. It is defined as "the synthesis, exchange and application of knowledge by relevant stakeholders to accelerate the benefits of global and local innovation in strengthening health systems and improving people's health. Knowledge translation is about creating, transferring and transforming knowledge from one social or organizational unit to another in a value-creating chain (Landry and Amara, 2006; Lee and Yang 2000). The transfer of knowledge from one community or organizational unit to another usually faces five problems: knowledge access, knowledge incompleteness, knowledge asymmetry, knowledge valuation and knowledge incompatibility. Knowledge translation is distinguished by at least three features:

a. Emphasizes the process of qualifying search evidence. b. Supports the flow of data and understanding from researcher to practitioner/ policy makers as well as the flow from practitioner/policy maker to researcher.

c. Pertains to complex social and organizational contexts.

While the term knowledge translation may appear similar to dissemination or diffusion, it can be differentiated by its emphasis on the quality of research prior to dissemination and implementation of research evidence into a system. Unlike simple dissemination activities, knowledge translation requires coordination and process improvement amongst a complex system. Knowledge translation involves the reporting, quality assessment and adaptation of research and development knowledge into an understandable and contextually relevant form that reflects organization's research and development priorities and that will be utilized by and benefit practitioner and consumers.

\section{Importance of knowledge translation}

Knowledge translation has emerged in response to the gap between evidence based research and its use / implementation by various stakeholders change in behaviors is usually the ultimate goal, but in practice the impact of knowledge translation is often much more suitable and long term.

\section{Knowledge mapping and knowledge translation}

Knowledge mapping comprises a process, which provides an organization with a picture of the specific knowledge it needs in order to support its business processes. Knowledge mapping is an important practice consisting of survey, audit and synthesis. It aims to track the acquisition and loss of information and knowledge. It explores personal and group competencies and proficiencies. It illustrates or "maps" how knowledge flows throughout an organization. Knowledge mapping helps an organization to appreciate how the loss of staff influences intellectual capital, to assist with the selection of terms, and to match technology to knowledge needs and processes. We have got some key principles of knowledge mapping like:

(a) Understand that knowledge is transient.

(b) Explain the sanction, establish boundaries, and respect personal disclosures.

(c) Recognize and locate knowledge in wide variety of forms: tacit and explicit, formal and informal, codified and personalized, internal and external, short life cycle and permanent.

(d) Locate knowledge in processes, relationships, policies, people, documents, conversations, links and context, suppliers, competitors and customers.

(e) Be aware of organizational level and aggregation, cultural issues and reward systems, timeliness, sharing and value, legal process and protection (patents, trade secrets, trade marks, NDAs).

The internal knowledge mapping in a public health organization allows it to learn what it knows. It refers to the understanding and self awareness that an organization has with respect to its knowledge resources and their limitations. Internal knowledge is especially important because it is unique, specific to the organization, tacit and therefore difficult to reproduce by knowledge holders located outside the organization. On the other hand, external knowledge acquisition refers to a capability for external awareness, more specifically to the capacity for identifying and acquiring knowledge from external sources and making it suitable for subsequent use by the organization. Knowledge mapping and acquisition involve many specific capacities for example, locating, accessing, valuing, and filtering pertinent knowledge, extracting, collecting, distilling, refining, interpreting, packaging, and transforming the captured knowledge into usable knowledge; and transferring the usable knowledge within the organization for subsequent use in the problem solving. External knowledge may provide new ideas and contexts for benchmarking internal knowledge; this type of knowledge is more explicit and more costly to acquire but it is easily available from other similar public health organizations.

With the help of results of the knowledge mapping we can look into the knowledge gap that may exist between what a public health organization has to know to implement its mandates and what it currently knows and this information leads to one of three conclusions:

1. The organization has external knowledge gap if it does not know enough to implements its public health mandate. 
2. The organization has external knowledge gap if it knows less than what other public health organization know in order to implement similar mandate.

3. The organization has knowledge gap it knows enough to implement its mandate or if it knows more than other public health organizations know in order to implement similar mandates.

Knowledge mapping may rely on one of four organizational modes: undirected viewing, conditioned viewing, informal search and formal search. In undirected viewing, a public health professional is exposed to information, when he or she has no specific informational needs in mind. We can say this is an informal strategy that can be useful for the early detection of emerging problems. In conditioned viewing a public health professional directs his or her viewing on information regarding selected public health topics or issues. During the informal search process, a public health professional looks for information that will improve his or her understanding of a specific public health issue. Finally, in a formal search a public health professional engages in a systematic search for ideas, information and knowledge about a specific health issue. This last mode includes conducting systematic reviews and external surveys as well as training and hiring employees (in order to bring knowledge into the organization).

Knowledge creation is usually associated with research and development activities. Knowledge translation capability refers to the capacity to combine knowledge (tacit, internal and external) in order to develop new knowledge. It also includes activities such as solving a public health problem, devising a public health promotion strategy, discovering a pattern, developing a public health programme or intervention or conducting, monitoring, and evaluation activities. On the other hand, knowledge destruction frequently paves the way for knowledge creation and innovation. However, it is difficult to destroy old knowledge and replace it with the implementation of new knowledge (for example, replacing old clinical guidelines with new).

\section{KNOWLEDGE INTEGRATION}

Knowledge integration is the capacity to transform a public health organization's knowledge resources (tacit, explicit, individual, organizational, internal, external) into actionable knowledge by taking into account the organization's strengths, weaknesses and opportunities as well as threats to the organization.

Over the time, public health organizations learn to integrate the accumulated internal knowledge with knowledge acquired from other organizations or other external sources. Organizations integrate the knowledge accumulated over time, developing and delivering programmes, interventions and services using knowledge from external sources. However, integrating of raw knowledge into actionable knowledge is necessary but not sufficient to solve the public health problems; knowledge must also be shared and transferred. Knowledge sharing is more demanding than knowledge reporting. Reporting involves disseminating information through codified formats to target groups within a public health organization (Ipe, 2003). By contrast, sharing implies person-to-person interactions during which one individual convert his or her (individual and often tacit) knowledge into a form that can be understood by other members in the organization. Knowledge sharing provides the mechanism to transform individual knowledge into organizational knowledge that can be redeployed to create value and solve problems at the organizational level. Knowledge sharing is a social process that may lead to the emergence of communities of practice. In public heath, such communities exist at the local, regional, national and international levels. We can say knowledge transfer complements knowledge sharing. While knowledge sharing is associated with an exchange of knowledge between individuals and knowledge exchange is associated with exchange knowledge between organizations or departments or divisions within the organizations. Knowledge that has been shared or transferred provides a template or guideline for decisions and actions. Knowledge replication is the capacity to identify the attributes of the knowledge that are replicable, how these attributes can be recreated and the characteristics of the contexts in which they can be replicated. But it is found that there are significant differences between the attributes of the knowledge and the contexts of the action and decisions described in the templates and guidelines, and a real public health context. So, knowledge replication must be guided by the attributes of the local context of actions and decisions, especially with respect to public health. The capacity to replicate knowledge improves the efficacy and efficiency of public health programmes and interventions. However, many legal mechanisms of knowledge protection like patent, copyright, trademarks etc. restrict use of knowledge replication. To what extent knowledge replication has been able to give desired results and outputs, it should be assessed by knowledge performance. Assessments are based on perspectives like:

(a) Value for money: investments in the creation, sharing and application of knowledge providing public health benefits.

(b) Knowledge users: the extent to which evidence based professional practices contributes to the development of new products and services to improve them.

(c) Final beneficiaries of knowledge translation: the extent to which evidence based professional practices are translated into new improved products and services.

(d) Internal organizational process: public health organizations must have an account of processes to achieve superior knowledge sharing, transfer, replication 
in order to achieve superior outcomes for the public.

\section{Conclusion}

The gap between what we know and what we practice, that is, know-do gap exist in public health organizations. Knowledge translation is also on important measure to bridge the gap between knowledge and action for health. It is hoped that public health organizations in developing countries will try to invest more resources in prompting measures like- knowledge translation, knowledge management to narrow the gap as well as develop a culture where decisions taken by policy-makers, health professional and the public are based on evidence.

\section{REFERENCES}

Ebener S, Khan A (2006). Knowledge mapping as a technique to support knowledge. Bulletin of the World Health Organization. 84(8): 636-42.

Ipe M (2003). Knowledge sharing is organizations; a conceptual framework. Human Res. Dev. Rev. 2:227-59.

Landry R, Amara N (2006). The knowledge - value chain: a conceptual framework for knowledge translation in India. Bull. World Health Organ. 84(8):597-602.

Lee CC, Yang J (2000). Knowledge - value chain. J. Manag. Dev. 19:783-793.

Vestal, APQC (2002). http://bit.ly/xrBURB. 